\title{
TaiChi and Qigong for Depressive Symptoms in Patients with Chronic Heart Failure: A Systematic Review with Meta-Analysis
}

\author{
Wei Jiang, ${ }^{1,2}$ Shaojun Liao $\mathbb{D}^{3}{ }^{3}$ Xiankun Chen $\mathbb{D}^{\mathrm{D}},{ }^{2,4,5}$ Cecilia Stålsby Lundborg, ${ }^{4}$ \\ Gaetano Marrone, ${ }^{4}$ Zehuai Wen $\mathbb{D}^{5,},{ }^{5,6}$ and Weihui Lu $\mathbb{D}^{1,2}$ \\ ${ }^{1}$ Department of Cardiology, Guangdong Provincial Hospital of Chinese Medicine, Guangzhou 510120, China \\ ${ }^{2}$ The Second Affiliated Hospital of Guangzhou University of Chinese Medicine, Guangzhou 510405, China \\ ${ }^{3}$ Second Clinical Medical College, Guangzhou University of Chinese Medicine, Guangzhou 510405, China \\ ${ }^{4}$ Health Systems and Policy, Department of Global Public Health, Karolinska Institutet, Stockholm 17177, Sweden \\ ${ }^{5}$ Key Unit of Methodology in Clinical Research, Guangdong Provincial Hospital of Chinese Medicine, Guangzhou 510120, China \\ ${ }^{6}$ National Centre for Design Measurement and Evaluation in Clinical Research, Guangzhou University of Chinese Medicine, \\ Guangzhou 510405, China
}

Correspondence should be addressed to Zehuai Wen; wenzehuai@139.com and Weihui Lu; weihui.lu@163.com

Received 11 March 2021; Accepted 14 June 2021; Published 25 June 2021

Academic Editor: Parco M. Siu

Copyright (c) 2021 Wei Jiang et al. This is an open access article distributed under the Creative Commons Attribution License, which permits unrestricted use, distribution, and reproduction in any medium, provided the original work is properly cited.

Background. Depression is a debilitating comorbidity of heart failure (HF) that needs assessment and management. Along with mind-body exercise to deal with HF with depression, the use of TaiChi and/or Qigong practices (TQPs) has increased. Therefore, this systematic review assesses the effects of TQPs on depression among patients with HF. Methods. Randomized controlled trials (RCTs) that examined the effect of TQPs on depression in patients with HF were searched by five databases (PubMed, Cochrane Central Register of Controlled Trials (CENTRAL), EMBASE, CINAHL, and China National Knowledge Infrastructure (CNKI)). With standardized mean difference (SMD) and 95\% confidence intervals (95\% CI), random-effects meta-analyses of the effect of TQPs on depressive symptoms were performed. Results. Of eight included RCTs, seven (481 patients) provided data for the metaanalysis. The pooling revealed that TQPs contribute to depression remission in HF (SMD $-0.66 ; 95 \%$ CI -0.98 to -0.33 , $\left.P<0.0001 ; I^{2}=64 \%\right)$. Its antidepressive effect was not influenced by intervention duration or exercise setting, but rather by ejection fraction subtype, depressive severity, and depression instruments. The beneficial effects were preserved when the study with the largest effect was removed. Conclusion. This study suggests that TQPs might be a good strategy for alleviating depressive symptoms in patients with HF. And rigorous-design RCTs, which focus on the identified research gaps, are needed to further establish the therapeutic effects of TQPs for depression in HF.

\section{Introduction}

Depression is a frequent comorbidity in patients with heart failure (HF) [1]. Data from meta-analysis have shown that patients with HF have a mean depression prevalence of $21.5 \%, 2$ to 3 times the rate of the general population [2]. Depression often exacerbates symptoms of patients with $\mathrm{HF}$, doubling the risk of mortality and other cardiac events among patients with HF [3]. It is also a strong predictor of future cardiac events related to $\mathrm{HF}$, similar to traditional cardiovascular risk factors [4]. Therefore, routine screening for and treatment of depression is an urgent need for patients experiencing HF. It is also suggested by the European Society of Cardiology (ESC) and American College of Cardiology/American Heart Association (ACC/AHA) HF guidelines $[5,6]$.

Review articles are now suggesting the therapeutic benefits of mind-body exercise on depression of varying severity [7-11]. Moreover, for patients with HF experiencing depression, interventions designed to improve both physical and psychological symptoms have been shown to reduce depression, increase physical function, and improve quality 
of life [12]. Thus, mind-body exercise may be the optimal therapy for depressive patients with HF.

TaiChi and Qigong are common mind-body exercises that originated in China. Each is an ancient Chinese healing art with a history of thousands of years [13, 14]. Qigong is an umbrella term covering a spectrum of mind-body exercises, such as Dao-Yin-Shu (physical and breathing exercise), WuQin-Xi (five-animal play), Ba-Duan-Jin (eight-section health exercise), and Yi-Jin-Jing (changing tendons exercise) [15]. It is characterized by simple physical movements and is thought to be the grandfather to TaiChi, while TaiChi derives from Qigong and Martial Arts, involving more complex and choreographed movements [16]. Both TaiChi and Qigong employ slow and gentle physical movements, synchronized with breathing regulation and meditation to stretch the body, relax the muscles, coordinate breathing, and regulate attention and consciousness $[17,18]$. Traditional Chinese medicine physicians believe that performing the meditative and synergistic dance-like movements of TaiChi and Qigong can promote Qi to flow harmoniously, thus promoting health and spirit [19].

Review articles and meta-analyses have demonstrated TaiChi's and Qigong's benefits on depression in the general population $[10,20]$, patients with chronic illnesses $[9,21-23]$, and patients with cardiovascular disease [24, 25]. Of note, TaiChi has served as a treatment for depression in many clinical trials, and evidence has shown that it is correlated with significant reductions in depression [18]. However, analysis for each illness has been limited to a handful of studies, precluding robust testing of whether the effects of TaiChi and Qigong differ by illness.

In recent years, many randomized controlled trials (RCTs) have reported the effects of TaiChi and Qigong among patients with HF, some of them reporting depression outcomes [26-33]. However, the results have been inconclusive, and convincing quantitative evidence to estimate treatment effects has been lacking. Therefore, this review evaluates the evidence from RCTs of TaiChi and Qigong on depression in patients with HF.

\section{Methods}

This systematic review was conducted and reported in accordance with the Preferred Reporting Items for Systematic Reviews and Meta-Analyses (PRISMA) statement [34] and the Cochrane Handbook for Interventional Reviews [35]. The study protocol has been published in PROSPERO (CRD42018081982). We followed the methods of Chen et al. (2020) [36].

2.1. Search Strategy. PubMed, Cochrane Central Register of Controlled Trials (CENTRAL), EMBASE, CINAHL, and China National Knowledge Infrastructure (CNKI) were searched from inceptions until October 23, 2019, without language restriction. Search terms were classified in three groups (Appendix 1: search strategy): condition (CHF, chronic heart failure and related terms), intervention (tradition Chinese exercise and related terms such as traditional exercise, mind-body exercise, Qigong, Taiji, Tai ji, Tai Chi, TaiChi, Liuzijue or Liu Zi Jue, or Baduanjin or Ba duan jin, or Wuqinxi or Wu Qin Xi, or Yijinjing, or Yi Jin Jing), and study type (RCT and related terms).

2.2. Study Selection. We applied the following selection criteria: (1) study design: RCTs reported in a full text; (2) participants: patients diagnosed with HF with or without restriction of left ventricular ejection fraction and in a stable phase of the disease; (3) interventions and control: RCTs comparing TaiChi and/or Qigong (TQPs) plus routine management (RM) with RM alone, or comparing TQPs plus RM with general exercise plus RM. RM included standard medical treatment, education, health guidance, or aerobic exercise. RCTs in which the intervention group was TQPs plus other traditional Chinese medicine therapies, such as acupuncture, Chinese herbal medicine preparation, were excluded; and (4) outcomes: depression or depressive symptoms measured by using patient-reported outcomes (PROs) or non-PRO assessment instruments.

After screening titles and abstracts from articles found in the searches, two reviewers (WJ, SL) retrieved potentially relevant full-text articles. These two reviewers independently assessed the eligibility of the full-text articles according to the selection criteria. Meanwhile, they resolved any discrepancies by agreement after rechecking the source articles or/and after discussion with a third reviewer (XC).

2.3. Data Extraction. One reviewer (WJ) extracted data with a standardized form from the included articles. A second reviewer (SL) checked the extracted data. Discrepancies were resolved by consensus after rechecking the source papers and further discussion with a third reviewer (XC).

The extracted data including study characteristics (e.g., title, author, publication year, and the use of randomization, allocation concealment, blinding and control, and country), participant characteristics (e.g., age, sex, sample size, New York Heart Association (NYHA) classification, and left ventricular ejection fraction (LVEF)), description of interventions (e.g., types of exercises, frequency, and durations) and controls, and instruments used to measure depression or depressive symptoms. Attempts were made to contact the original investigators regarding any missing data.

2.4. Risk of Bias Assessment. The trials' methodological quality was independently evaluated by two reviewers (WJ and SL) using the Cochrane risk of bias assessment tool. Discrepancies were resolved by agreement after rechecking the source papers and further discussion with a third reviewer (XC). Two reviewers assessed a quality rating for the following domains for each included trial as high risk, low risk, or uncertain risk of bias: (1) random sequence generation; (2) allocation concealment; (3) blinding of participants and personnel; (4) blinding of outcome assessors; (5) incomplete outcome data; and (6) selective reporting. 
2.5. Data Analysis. The data from the included studies were analysed with RevMan 5.3 and STATA 12 . Heterogeneity was assessed using a chi-square test (a $P$ value $<0.10$ was considered indicative of statistical significance) and an $I^{2}$ statistic (where $I^{2}>30 \%, 50 \%$, or $75 \%$ indicated moderate, substantial, and considerable heterogeneity, respectively). Then, data from each trial were pooled with a random effects model in order to provide the included studies with more uniform weight. Given that all variables in the included studies consisted of continuous data and that various instruments were used, we used standardized mean difference (SMD) with 95\% confidence intervals (95\% CI) to analyse the outcomes. For studies with more than 2 control groups [33], such as TQPs plus RM vs. general exercise plus RM vs. RM alone, the means and standard deviations (SDs) of the two controls were combined using the methods described in the Cochrane Handbook (Section 6.5.2.10). A $P$ value $<0.05$ was considered statistically significant.

Sensitivity analysis was first conducted by removing each study individually to estimate the results' consistency, as well as to explore the heterogeneity contributed from each individual study. Thereafter, subgroup analysis was undertaken to investigate the role of the various study characteristics on the observed effect, as well as to identify any potential sources of heterogeneity [37].

For trials with missing information on the means or SDs of outcomes, data were first sought from the original investigators. If it was not available from the author, then imputations were performed using the statistical approaches recommended in the Cochrane Handbook. One study used the median and interquartile ranges (IQR) instead of means and SDs [31]; the medians were taken as a substitute for the means and the $\mathrm{SD}$ was approximated as $\mathrm{SD}=\mathrm{IQR} / 1.35$. In another study, SDs were imputed from a reported confidence interval [28] with missing SDs. Publication bias was not assessed due to the limited number of studies $(<10)$ included in each analysis.

\section{Results}

1480 records (PubMed $(n=31)$, EMABSE $(n=64)$, Cochrane $(n=156)$, CINAHL $(n=29)$, CNKI $(n=1191))$ totally were retrieved from the database searches. After excluding duplicates, 999 potentially relevant abstracts were screened, and 934 were excluded for failing to meet the inclusion criteria. The remaining 65 full texts were read, and finally, 8 RCTs [26-33] were deemed eligible for this review. Figure 1 presents reasons for exclusion.

\subsection{Characteristics of the Included Studies}

3.1.1. Study Characteristics. Table 1 shows the main characteristics of selected studies. Eight RCTs (3 in Chinese [26-28] and 5 in English [29-33]) were published between 2007 and 2019. Regions of publication were the United States $(n=4)$ [30-33], Mainland China $(n=2)$ [26, 27], Taiwan $(n=1)$ [28], and the United Kingdom $(n=1)$ [29].
3.1.2. Participants. Sample size per RCT ranged from 16 to 113 , with a total of 514 patients who were elderly (mean age: 65 to 68 yrs.). The percentage of males ranged from $50 \%$ to $88 \%$. Most patients were in NYHA classes II and III, and with LVEFs $<40 \%$, while two trials included patients with LVEFs $\geq 40 \%[32,33]$.

3.1.3. Intervention and Control. The majority of the trials used TaiChi $(n=6)[26,27,30-33]$, and the rest used Qigong (Chan-Chuang) [28] or TaiChi plus Qigong [29]. Exercise times lasted from 15 to 60 minutes per session. The length of exercise programs was either 12 weeks $(n=5)$ $[26,28,30-32], 16$ weeks $(n=2)[29,33]$, or 24 weeks $(n=1)$ [27]. The exercises were center-based in 5 trials [29-33], and home-based in 3 trials [26-28]. The controls received the typical care, including medication and education advice in all trials and also received formal aerobic exercise training [32] or resistance band exercise [33].

3.1.4. Outcome Measurement Instruments. The included studies used various depression severity scales (Table 2). This included three depression specific scales: the Hamilton Rating Scale for Depression (HAM-D) [26, 27], the Beck Depression Inventory (BDI) [30-33], and the Hospital Anxiety and Depression Scale (HADS) [28]. The HAM-D was assessed by clinicians while BDI and HADS were selfrated scales. Two general instruments were used and depression was reported as subscale scores: the Symptom Checklist 90-Revised (SCL-90R) [29] and the Profile of Mood States (PMOS) full [31] and brief versions [32]. Both instruments were self-rated scales.

3.1.5. Depression Status. Based on the baseline mean scores and the reference cutoff points of the depression severity scales (Tables 1 and 2), participants were mildly depressed in four studies [28-30,33] and moderately depressed in two studies $[26,27]$. The other two studies using PMOS reported that $30 \%$ [31] and 37\% [32] of the subjects had depression as a comorbidity, respectively. However, it was difficult to determine the average severity of the included participants as neither the classification nor the cutoff points of the PMOS scale were reported. Only one study applied clinically diagnosed depression as inclusion criteria for participants [26].

3.2. Methodological Quality of the Evidence. Table 1 presents the risk of bias assessments for individual studies (Appendix 3 : risk of bias analysis). Five [26, 28, 31-33] out of eight RCTs described the methods of randomization. However, only two of the included trials $[28,33]$ reported allocation concealment details. Blinding of participants and personnel were judged as high risk of bias for most of the trials [26-30] due to the nature of the intervention. Blinding of outcome assessors was judged as high risk of bias for patient-reported scales. Two [26, 27] claimed that statisticians had been blinded. Most of the articles showed a low risk of incomplete outcome bias [26, 27, 29, 31-33]. Selective reporting bias was 


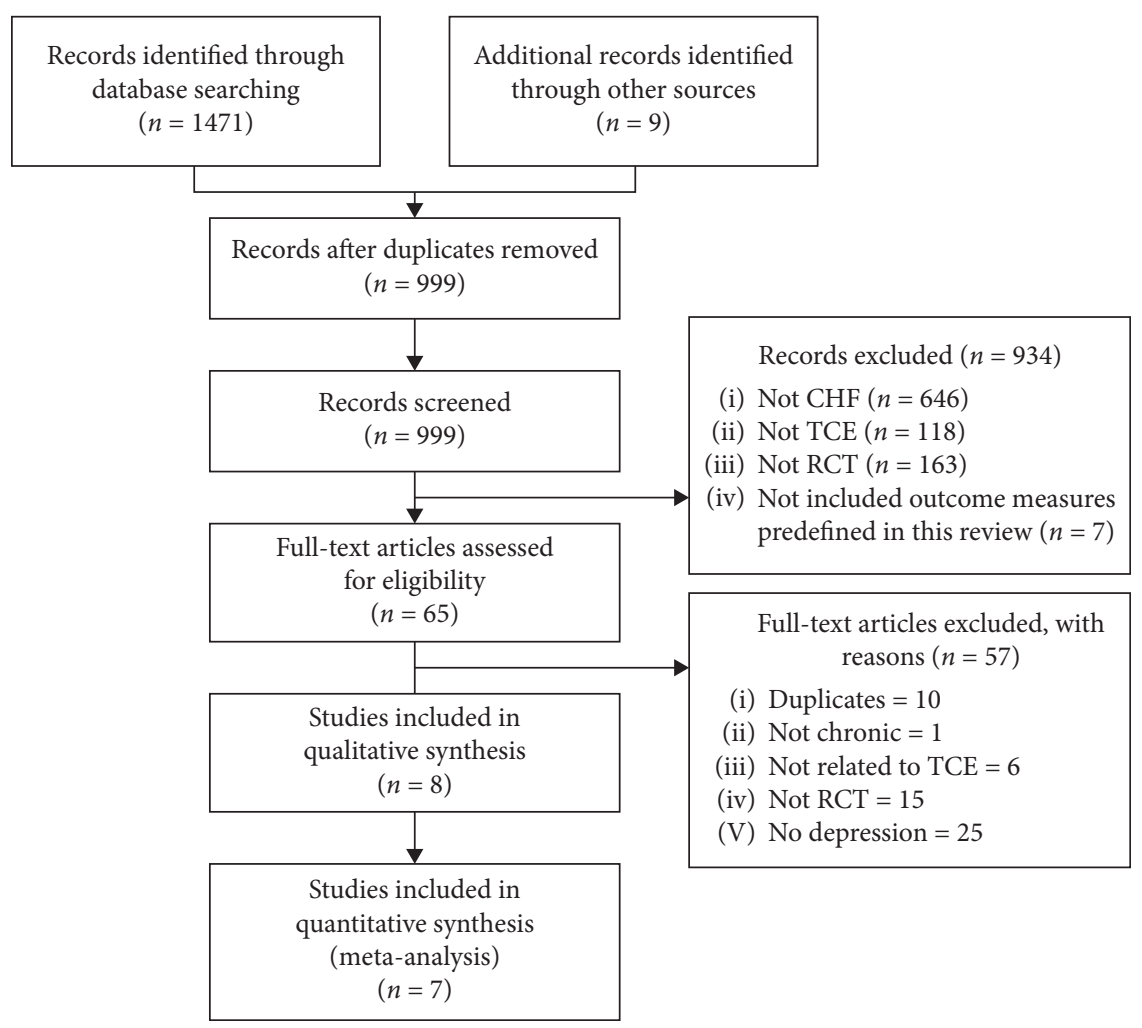

FIGURE 1: Search strategy and flow chart of the screened, excluded, and analysed articles. CHF: heart failure, TCE: traditional Chinese exercise, and RCT: randomized controlled trial.

unclear in most RCTs [26-31, 33] because neither protocol nor trial registration information was available.

3.3. Outcomes. One study, conducted by Redwile et al. (2012) [30], could not be incorporated into the meta-analysis because of the incomplete data presented. This study included 28 elderly patients (mean age 68 yrs.) undertaking 12 week center-based TaiChi training $(n=16)$ or typical care $(n=12)$. The author reported that patients in the TaiChi group had experienced reduced BDI total symptom scores from pre- to postintervention, compared to the controls. However, depression values were only provided at baseline, but not at the $12^{\text {th }}$ week. The remaining 7 trials reported various outcomes, including measurements of symptoms and depression status, which were included in the quantitative synthesis.

3.3.1. Overall Effects. Pooling across the remaining seven RCTs (481 patients) provided evidence of a decrease in the depression symptoms with TQPs, but this analysis demonstrated substantial heterogeneity (SMD $-0.66,95 \% \mathrm{CI}$ -0.98 to $-0.33, P<0.0001 ; I^{2}=64 \%$; Figure 2 ; Appendix 2 : subgroup analysis (overall pooled effect)). Here a sensitive analysis was performed with one study removed at a time to explore potential sources of heterogeneity, as well as to assess whether the result could have been affected markedly by a single study. The result showed that Yeh et al.'s (2011) study [31] was removed, the statistical heterogeneity disappeared, and the pooled results continued to significantly favor the TQPs with smaller effects (SMD $-0.54,95 \%$ CI -0.74 to $-0.33, P<0.00001 ; I^{2}=0 \%$; Figure 2; Appendix 2: subgroup analysis (overall pooled effect)). However, omitting other studies altered neither effect estimates nor heterogeneity (Figure 3).

3.3.2. Subgroup Analysis. We conducted subgroup analysis to investigate the role of the various study characteristics on the pooled effects, as well as to provide estimates of treatment effects for clinically relevant subgroups.

(1) Participants' EF. The benefits of TQPs on depressive symptoms according to ejection fraction (EF) subtype were inconsistent (Figure 4(a); Appendix 2: subgroup analysis (EF subtypes)). The pooled results favored TQPs with increased effect size and remained significant for the heart failure with reduced ejection fraction (HFrEF) RCTs. However, when pooling the two trials including patients with both $\mathrm{HFrEF}$ and heart failure with preserved ejection fraction (HFpEF), an insignificant beneficial effect towards the TQPs was found.

(2) Depressive Severity. In the subgroup analysis for participants' depressive severity, the moderately depressed patients benefited more than the mildly depressed patients (SMD -0.76 versus -0.37; Figure 4(b); Appendix 2: subgroup analysis (depressive severity)). However, when pooling the two trials with unknown depression severity, the beneficial effects became insignificant. 


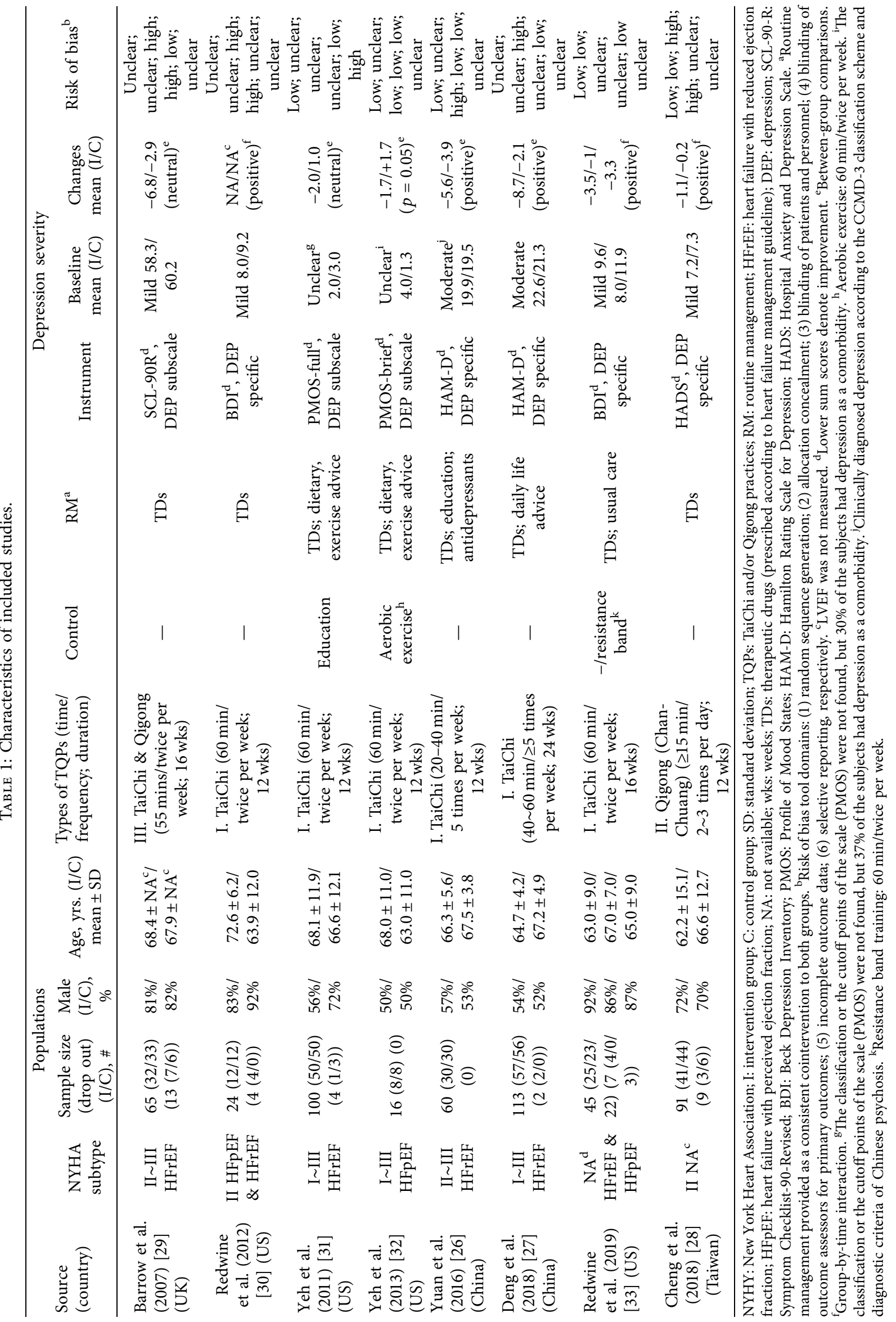


TABLE 2: Summary of the depression severity scales used in the included studies.

\begin{tabular}{|c|c|c|c|}
\hline $\begin{array}{l}\text { Instruments } \\
\text { (no. of study) }\end{array}$ & Objective & $\begin{array}{l}\text { Rater; number of item; rating } \\
\text { scale }\end{array}$ & Categorization/cutoff \\
\hline $\begin{array}{l}\text { SCL-90R, DEP } \\
\text { subscale }(n=1)[29]\end{array}$ & $\begin{array}{l}\text { To reflect the psychological symptom patterns in } 9 \\
\text { domains: somatization/obsessive-compulsive/ } \\
\text { sensitivity/depression/anxiety/hostility/phobic } \\
\text { anxiety/paranoid ideation/psychoticism }\end{array}$ & $\begin{array}{l}\text { PRO; } 90 \text { items (DEP: } n=13) \\
\text { 5-point scale }(0 \sim 4)^{\mathrm{a}}\end{array}$ & $\begin{array}{l}\text { A } \mathrm{T} \text {-score }{ }^{\mathrm{b}} \text { ranging from } 40 \text { to } \\
60 \text { represents the normal range }^{\mathrm{c}}\end{array}$ \\
\hline $\begin{array}{l}\text { BDI, DEP specific } \\
(n=2)[30,33]\end{array}$ & $\begin{array}{c}\text { To measure the severity of depression in adults } \\
\text { and adolescents, two subscales include a } \\
\text { cognitive-affective subscale and a somatic- } \\
\text { performance subscale }\end{array}$ & $\begin{array}{l}\text { PRO; } 21 \text { items; 4-point scale } \\
(0 \sim 3)^{\mathrm{a}}\end{array}$ & $\begin{array}{l}\text { 0-13: minimal; 14-19: mild } \\
\text { depression; 20-28: moderate; } \\
\text { 29-63: severe }{ }^{\mathrm{d}} \\
\text { In nonclinical populations, } \\
\text { scores above } 20 \text { indicate } \\
\text { depression }\end{array}$ \\
\hline $\begin{array}{l}\text { PMOS-full, DEP } \\
\text { subscale }(n=1)[31]\end{array}$ & $\begin{array}{c}\text { To assess emotional states in } 6 \text { domains: } \\
\text { depression/anxiety/fatigue/vigor/irritability/ } \\
\text { confusion }\end{array}$ & $\begin{array}{l}\text { PRO; } 65 \text { items (DEP: } n=15) \\
\text { 5-point scale }(0 \sim 4)^{\mathrm{a}}\end{array}$ & Not found \\
\hline $\begin{array}{l}\text { PMOS-brief DEP } \\
\text { subscale }(n=1)[32]\end{array}$ & Same as the full version & $\begin{array}{l}\text { PRO; } 30 \text { items (DEP: } n=5) \\
\text { 5-point scale }(0 \sim 4)^{\mathrm{a}}\end{array}$ & Not found \\
\hline $\begin{array}{l}\text { HAM-D DEP } \\
\text { specific }(n=2) \\
{[26,27]}\end{array}$ & $\begin{array}{l}\text { The "gold standard" for assessing severity of } \\
\text { depressive severity }\end{array}$ & $\begin{array}{l}\text { Clinician; } 17 \text { items; } 5 \text {-point } \\
\text { scale }(0-4)^{\mathrm{a}}(n=8) ; 3 \text {-point } \\
\quad \text { scale }(0-2)^{\mathrm{a}}(n=9)\end{array}$ & $\begin{array}{l}0-7: \text { normal; } 8-16: \text { mild; } 17-23: \\
\text { moderate; } 24-50: \text { severe }^{\mathrm{e}}\end{array}$ \\
\hline $\begin{array}{l}\text { HADS DEP specific }{ }^{\mathrm{f}} \\
(n=1)[28]\end{array}$ & $\begin{array}{l}\text { To assess anxiety and depression symptoms in } \\
\text { medical patients }\end{array}$ & $\begin{array}{l}\text { PRO; } 14 \text { items (DEP: } n=7) \\
\text { 4-point scale }(0 \sim 3)^{\mathrm{a}}\end{array}$ & $\begin{array}{l}0-7 \text { : normal; } 8-10: \text { mild; } 11-14: \\
\text { moderate; } 15-21: \text { severe } \\
\text { A cutoff of } 8: \text { clinically } \\
\text { significant depression }\end{array}$ \\
\hline
\end{tabular}

PRO: patient-reported outcome; DEP: depression; SCL-90R: Symptom Checklist-90-Revised; BDI: Beck Depression Inventory; PMOS: Profile of Mood

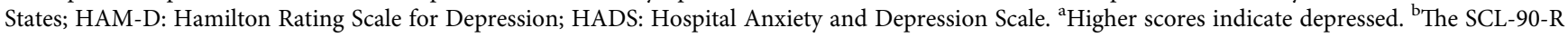
scores are converted to standard $T$-scores (ranging from 30 to 80 ) by referring to the appropriate population-based norm tables provided by the test manual and a $T$-score of 50 represents the mean of the respective normal population. ${ }^{c} \mathrm{Holi}, \mathrm{M}$. (2003). Assessment of psychiatric symptoms using the SCL-90. ${ }^{\mathrm{d}}$ Jackson-Koku, G. (2016). Beck depression inventory. Occupational Medicine, 66 (2), 174-175. ' Zimmerman, M., Martinez, J. H., Young, D., Chelminski, I., \&

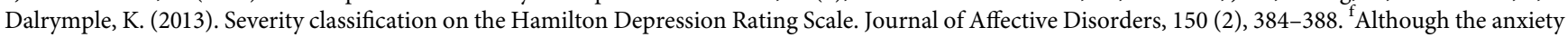
and depression questions are interspersed within the questionnaire, it is vital that these are scored separately. ${ }^{\mathrm{g}}$ Stern, A. F. (2014). The Hospital Anxiety and Depression Scale. Occupational Medicine, 64 (5), 393-394.

(3) Depression Instruments. The pooled results were inconsistent across subgroups according to the depression instruments (Figure 4C; Appendix 2: subgroup analysis (depression instrument)). When pooling two trials in which the depression severity was evaluated by the clinicians, the beneficial effects from TQPs exceeded the overall effects (Figure 4(c): clinician; Appendix 2: subgroup analysis (depression instrument)). However, when pooling two trials in which patients self-rated their depressive severity using depression specific instruments (BDI and HADS), the beneficial effects from TQPs became smaller and insignificant (Figure 4(c): PRO/specific; Appendix 2: subgroup analysis (depression instrument)). Of note, when pooling the other three trials in which depressive severity was also self-rated by patients, but using nondepression specific instruments (PMOS and SCL-90R), the pooled results became significant again with a larger effect (Figure 4(c): PRO/nonspecific; Appendix 2: subgroup analysis (depression instrument)).

(4) Characteristics of TQP Programs. Generally, the pooled effects were not influenced by the characteristics of the TQP programs. There were similar pooled effect sizes in the TaiChi subgroup (Figure 4(d); Appendix 2: subgroup analysis (TQPs)). In addition, TQP program length $(<12$ weeks vs. $>12$ weeks) might not have influenced the pooled effect sizes (Figure 4(e); Appendix 2: subgroup analysis (length of TQP programs)). Furthermore, the effect sizes were also similar whether the TQPs were delivered at centers or were home-based (Figure 4(f); Appendix 2: subgroup analysis (TQP delivery settings)). As the sensitivity analysis showed that the study by Yeh et al. (2011) [31] was the main contributor to the heterogeneity (Figure 3), we removed this study and then repeated the subgroup analysis. This change solved the statistical heterogeneity and resulted in a mitigated pooled effect, but did not alter the (in)significance of the pooled results in the associated subgroups (dashed line in Figure 4).

3.4. TQP Safety and Overall Evidence. Patient dropout in the TQP groups was low, with most withdrawals being due to hospitalization or CHF exacerbation. We found no adverse events related to TQPs in the included studies.

\section{Discussion}

To our knowledge, this is the first systematic review to synthesize RCTs written in both Chinese and English languages that have explored the potential effects of TaiChi and Qigong on depression among patients with HF. Eight RCTs were included, and seven RCTs involving 481 patients with HF were evaluated in the meta-analysis. The results showed that practicing TaiChi and Qigong were associated with a significant reduction in depressive symptoms (SMD -0.66, $95 \%$ CI -0.98 to $-0.33, P<0.0001 ; I^{2}=64 \%$ ), and its 


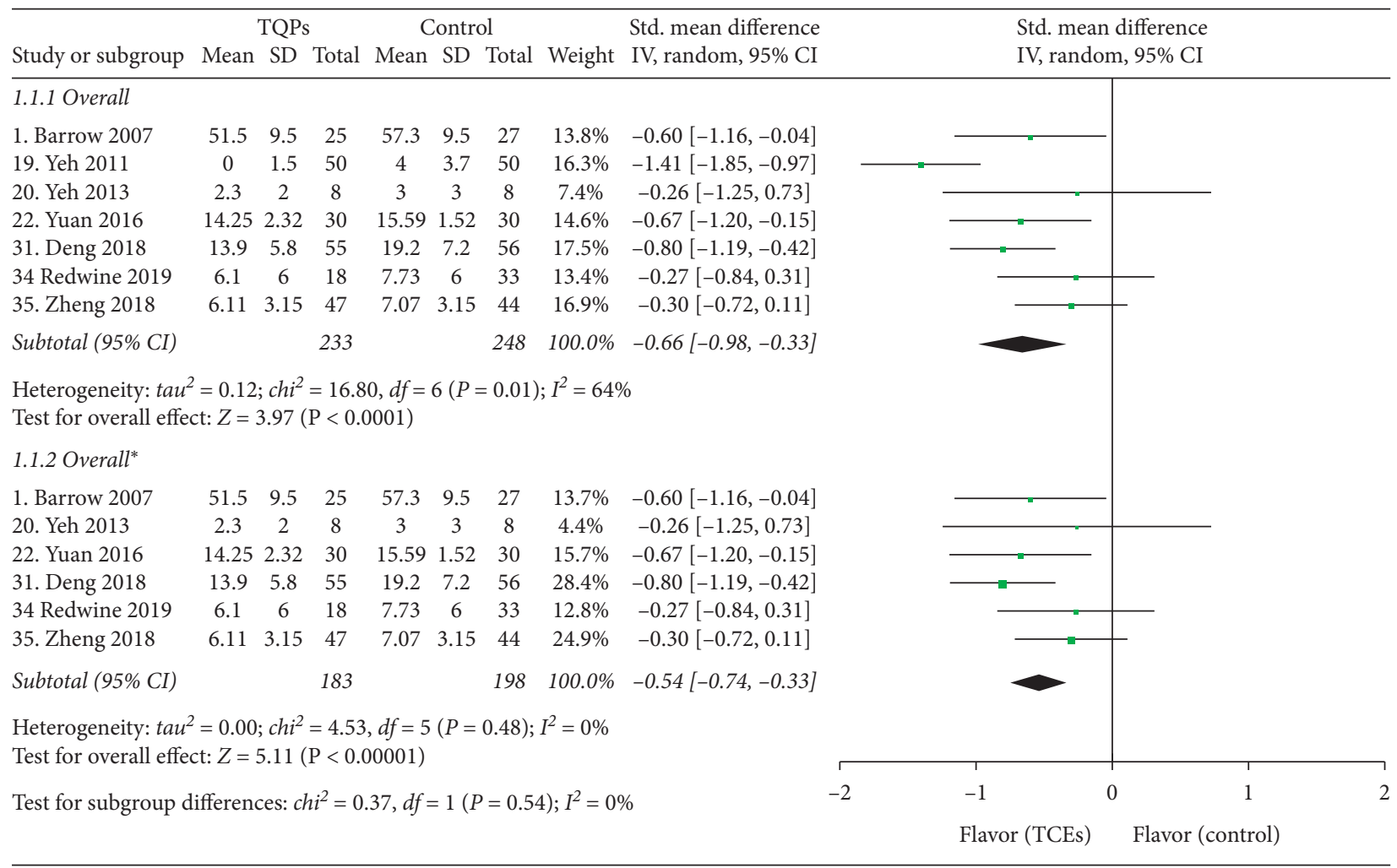

FIGURE 2: Meta-analysis results of overall pooled effects.

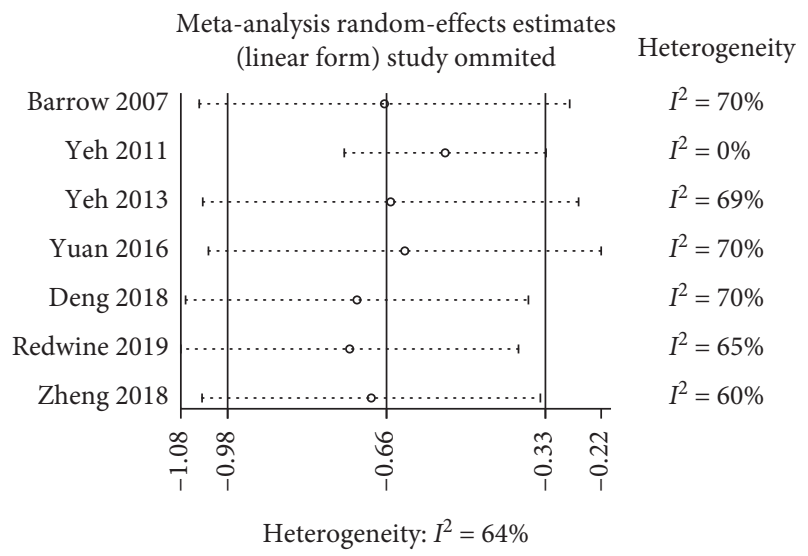

FIGURE 3: Influence of each individual study on the overall pooled effect estimate.

antidepressive effect was not influenced by intervention duration or exercise setting, but rather by EF subtype, depressive severity, and depression instruments. Significant effects were found for HFrEF (SMD: -0.89 ; 95\% CI -1.25 to $-0.53 ; I^{2}=54 \%$ ), moderate depression (SMD: $-0.76 ; 95 \% \mathrm{CI}$ -1.07 to $-0.45 ; I^{2}=0 \%$ ), mild depression (SMD: $-0.37 ; 95 \%$ CI -0.66 to $\left.-0.09 ; I^{2}=0 \%\right)$, specific clinician-rated scales (HAM-D) evaluated depression severity (SMD: $-0.76 ; 95 \%$ CI -1.07 to $-0.45 ; I^{2}=0 \%$ ), and nonspecific self-rated scales (PMOS and SCL-90R) evaluated depression severity (SMD: $-0.84 ; 95 \%$ CI -1.53 to $-0.15 ; I^{2}=73 \%$ ); however, not for HFrEF and HFpEF, specific self-rated scales (BDI and HADS) measured depressive severity. In addition, the beneficial effects of TQPs were preserved when we removed the study with the largest effect (SMD $-0.54,95 \% \mathrm{CI}-0.74$ to $\left.-0.33, P<0.00001 ; I^{2}=0 \%\right)$.

Our review is consistent with other systematic reviews and meta-analysis supporting the fact that TaiChi and Qigong reduce depressive symptoms [9, 10, 21]. One review of 17 randomized controlled or nonrandomized trials found that TaiChi reduced depression by heterogeneous standardized effects of -0.66 (95\% CI -0.29 to -1.03 ) among various populations [10]. Gu et al. [38] reviewed evidence on TaiChi in relation to various clinical outcomes including depressive symptoms among patients with $\mathrm{HF}$, founding that TaiChi resulted in significant depression-reduction effects, compared to the control. However, this result was based on only two RCTs involving 112 participants from the USA, most of whom were white. Unlike these previous studies, our review provides a synthesis of the evidence on all types of TaiChi and Qigong with a focus on patients with HF. In addition, previous studies have suggested that emotional responses to TaiChi or Qigong may vary across cultures [39]. Through extensive literature search, our review included the most updated evidence from Mainland China, Taiwan, the USA, and the UK, covering a range of ethnic groups.

The mechanism whereby traditional Chinese Qigong attenuates symptoms of depression is probably multifactorial. An underlying philosophy of the practice is that any form of traditional Chinese Qigong has an effect on the cultivation of balance and the harmony of vital energy (Qi), which functions as a holistic, coherent, and mutually interactive system [18]. In terms of biological mechanisms, it 


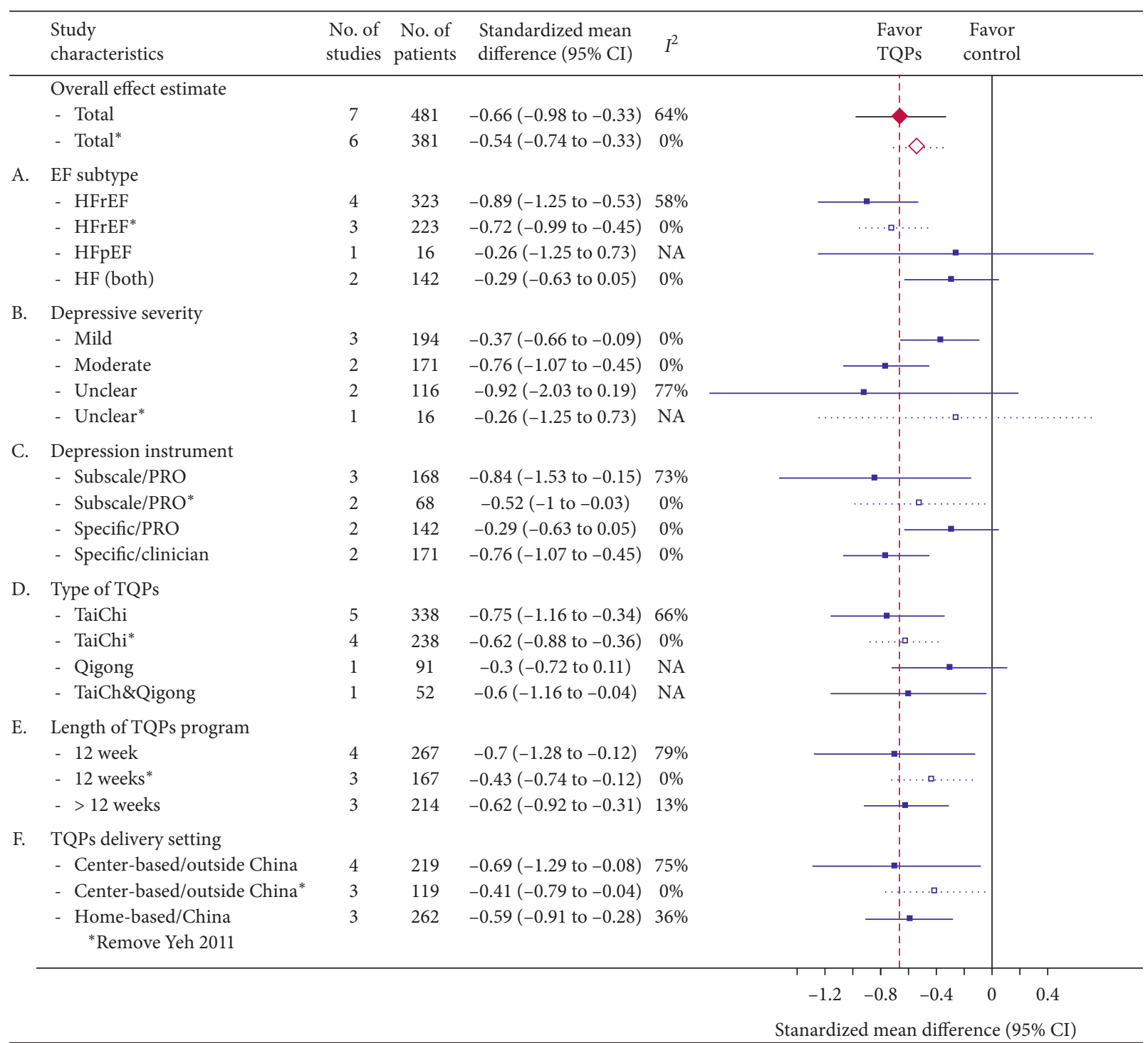

FIGURE 4: Influence of various study characteristics on the pooled effect and their contributions to heterogeneity. ${ }^{\mathrm{a}}$ Details of meta-analysis results showing individual study data are presented in Appendix 2.

has been reported that the activity and connectivity of key brain regions related to depression, the autonomic nervous system, and neuroinflammatory sensitization can be modulated by TaiChi and Qigong [18]. Another proposed mechanism suggests that the antidepressant effects of TaiChi and Qigong are associated with improvement in other clinical outcomes such as functional capacity and quality of life [18]. Measures of psychological variables and a multitude of other outcome measures are empirically interrelated, and treatment of each outcome can reciprocally and exponentially improve the other. Additionally, the mutual encouragement and friendly companionship from peers due to the collective activities of TQPs benefit the cognitive control network, adding to the effects related to the TaiChi or Qigong intervention [23].

4.1. Implications for Future Research. Although promising, several knowledge gaps identified in the present review need rational consideration and emphasis in future research. Data from the present review suggests favorable effects of TQPs on depressive symptoms among patients with HF, but this effect is restricted to depressed patients with HFrEF; it is not presented among depressed patients with HFpEF. The negative effects of TQPs on depressive symptoms in HFpEF remain to be determined because there was only one study [32] reviewed in our article regarding TQPs' effects on HFpEF, and it had a small sample size $(n=16)$. These findings accord with a previous meta-analysis assessing exercise training for patients with $\mathrm{HF}$ and comorbid depression in which only three trials were available regarding the antidepressive effect of exercise training on HFpEF [40].

There is no denying that insufficient samples partially explain the limited benefits experienced by patients with HFpEF and depression. Therefore, future large-scale multicenter RCTs with a sufficient number of participants are needed to verify TQPs' positive effects among this population. Likewise, the antidepressive effect of TQPs for 
patients with $\mathrm{HF}$ and comorbid major depression is less established. In our review, the available data describe the improvement due to TQPs in minor and moderate depression, and even depression without clear severity in patients with HF, except for major depression. Prior work [12] has emphasized that patients with HF combined with various depression severity have significant differences in the patterns of studies' findings of primary outcomes, such as depression, physical function, and quality of life. This suggests that the findings should be dichotomized according to depression severity to clarify the intervention effects. For this reason, it would be unreasonable to speculate TQPs' benefit on major depression based on its antidepressive effect on minor or moderate depression. Future studies need to enroll patients with HF whose major depression can be diagnosed definitively to evaluate TQPs' antidepressive effect.

In terms of TQPs' antidepressive effect, the present review indicates that it varies depending on the depression instruments used. In this light, it would be informative to consider whether and how to choose suitable depression instruments based on patient population, depression severity, and study setting when evaluating the intervention effects. Thus far, at least two instruments beyond those mentioned in our review have been widely applied to studies to detect and quantify depression in HF, i.e., the 9-item Patient Health Questionnaire (PHQ-9) [41] and the Montgomery and Asberg Depression Rating Scale (MADRS) [42]. However, many studies, including influential trials such as the HF-ACTION [43], the MOOD-HF [42], and the SADAHRT-CHF [44] do not state the usage reasons for a specific instrument. Thus, their results are less convincing. The 21-item Beck Depression Inventory (BDI-II) and the PHQ-9 are the more mature instruments and are recommended in HF combined with depression $[43,45,46]$. In view of a lack of specific guidelines for using depression instruments in HF, hopefully future clinical trials using BDIII and PHQ-9 will accurately measure depressive symptoms and disorders in patients with HF.

In addition, data indicated that Qigong, or TaiChi combined with Qigong, is less promising than TaiChi alone for depressed patients with HF. This leaves TaiChi as the state-of-the-art mind-body exercise for heart failure and depression in Chinese medicine. Yet definitive answers are missing, and the most effective type of intervention remains to be determined. According to our systematic review, to date there has only been one trial [28] with a small sample reporting changes in depressive symptoms with Qigong practice. This is insufficient to conclude that patients with HF suffering from depression do not respond well to Qigong. Moreover, the reduction in Qigong's comparative disadvantage to TaiChi was achieved indirectly by comparing to control groups. The results of this analysis are similar to another meta-analysis on traditional Chinese exercises (TCEs) for depression accompanying cardiovascular disease [24], which failed to investigate the benefits of specific TCEs to identify the ideal intervention. The situation for TaiChi and Qigong [29] is similar. In this light, there is an unmet need in HF for sufficiently powered trials of TQPs' effect on depression remission. Moreover, a larger-scale RCT on Qigong, an RCT to compare TCEs, and a network meta-analysis comparing TCEs could fill this research gap, thus determining the optimal TCEs to treat depression in HF.

Furthermore, data existing fails to demonstrate differences between the TQPs' effects compared with general exercise on depression in patients with HF. A recent systematic review and network meta-analysis on clinical depression in older adults [47] pointed out that mind-body exercise showed the largest improvement on depressive symptoms, followed by aerobic exercise and resistance exercise, despite a lack of statistically significant differences. Nevertheless, the results only provide limited insight into TQPs for depression in HF. In line with the present review, at this stage, we can only speculate on the relative benefits of TQPs based on two studies. These studies compared the antidepressive effect between TaiChi and aerobic exercise and between Qigong and resistance band training. Therefore, well-designed RCTs are needed to verify the relative benefits of TQPs for depression in HF to general exercise, including aerobic exercise and resistance exercise. This could promote a more solid foundation for treatment recommendations.

However, data from our review have demonstrated how TQPs mitigate depressive symptoms, but the benefits in clinical outcomes such as hospitalization and mortality remain uncertain. Logic would allow one to assume that treating depression may decrease poor outcomes in patients with $\mathrm{HF}$, given that depression can increase the risk of poor outcomes for patients experiencing HF. For example, the HF-ACTION trials [43] showed that exercise benefits patients with HF. Its ancillary study documented that reduced depressive symptoms were associated with improved clinical outcomes. This provided valuable insight into the screening and exercise treatment of depression in patients with HF. Thus, future studies should be designed to determine whether reduced depressive symptoms are associated with improved clinical outcomes, in addition to assessing the effects of TQPs on depressive symptoms.

4.2. Limitations. Several limitations in the present analysis should be noted. First, our analysis of the depression data symptoms was restricted to an SMD, as numerous depression scales were used in the included trials. Therefore, the results should be interpreted with this in mind. Another limitation is the presence of statistical heterogeneity between the trials in this meta-analysis. In order to provide the studies with more uniform weight, we used a random effects model. A sensitivity analysis and subgroup analysis were also performed to investigate the heterogeneity. In our metaanalysis, the beneficial effects [31] were preserved without heterogeneity when the study with the largest effect was removed. Finally, our conclusions were constrained by the quality of the trials reviewed. The main shortcomings were the lack of blinding procedures. However, in exercise interventions, double-blinding is not feasible without deception. In addition, most of the instruments used were 
self-rated scales where blinding the outcome assessors, i.e., the patients themselves, would have been impossible. These two features could have led to favorable responses among the participants in the intervention group. Therefore, blinding statisticians is essential in this type of trial, but only one included trial provided this information. The absence of randomization concealment and of the intention-to-treat (ITT) analyses is another concern when interpreting the results. Publication bias, although not evaluated, might have been present in the included studies since positive trials are more likely to be published than negative trials. Hence, the effect sizes might have been overestimated. Finally, many studies have neglected critical information in terms of allocation concealment, outcome assessor blinding, adequate follow-up, and ITT analysis. Future RCTs are warranted to improve the methodological quality by adhering to the Consolidated Standards of Reporting Trials statement (CONSORT) [48] or its extensions [49].

\section{Conclusion}

The evidence presented in this review should encourage physicians to recommend TQPs as clinically effective ways to reduce depressive symptoms in patients with HF. Additional RCTs with rigorous research design, which focus on the above research gaps, are warranted to establish the therapeutic effects of TQPs for depression in HF.

\section{Abbreviations}

BDI: Beck Depression Inventory

CI: $\quad$ Confidence intervals

EF: $\quad$ Ejection fraction

HADS: Hospital Anxiety and Depression Scale

HAM-D: Hamilton Rating Scale for Depression

HF: $\quad$ Heart failure

HFpEF: Heart failure with preserved ejection fraction

HFrEF: Heart failure with reduced ejection fraction

IQR: Interquartile ranges

ITT: Intention-to-treat

LVEF: Left ventricular ejection fraction

MADRS: Montgomery and Asberg Depression Rating Scale

NYHA: New York Heart Association

PHQ-9: 9-item Patient Health Questionnaire

PMOS: $\quad$ Profile of Mood States

PROs: $\quad$ Patient-reported outcomes

RCTs: Randomized controlled trials

RM: $\quad$ Routine management

SCL- Symptom Checklist 90-Revised

90R:

SDs: $\quad$ Standard deviations

SMD: $\quad$ Standardized mean difference

TCEs: Traditional Chinese exercises

TQPs: TaiChi and/or Qigong practices.

\section{Data Availability}

The extracted data used to support the findings of this study are available from the corresponding author upon request.

\section{Disclosure}

This study was part of a $\mathrm{PhD}$ thesis supported by the China Scholarships Council (201608440264).

\section{Conflicts of Interest}

All authors declare that there are no conflicts of interest regarding the publication of this paper.

\section{Authors' Contributions}

WJ and SL contributed equally to this work, conducted the main analysis, and drafted this manuscript. WJ and XC designed the research. GM, ZW, and WL joined in the discussion and offered interesting ideas. CL assisted in the preparation of the research. ZW and WL reviewed the text and guided the structure of the manuscript. All authors took part in the research and wrote, read, and approved the manuscript.

\section{Acknowledgments}

This work was supported by the General Research Fund of Traditional Chinese Medicine Science and Technology from the Guangdong Provincial Hospital of Chinese Medicine (YN2018ML02) and the Clinical Research Fund of the Traditional Chinese Medicine Science and Technology (Project 1010) from Guangdong Provincial Hospital of Chinese Medicine (YN10101910).

\section{Supplementary Materials}

Appendix 1: search strategy. Appendix 2: subgroup analysis. Appendix 3: risk of bias analysis. Appendix 4: PRISMA 2009 checklist. (Supplementary Materials)

\section{References}

[1] K. E. Di Palo, "Psychological disorders in heart failure," Heart Failure Clinics, vol. 16, no. 1, pp. 131-138, 2020.

[2] T. Rutledge, V. A. Reis, S. E. Linke, B. H. Greenberg, and P. J. Mills, "Depression in heart failure," Journal of the American College of Cardiology, vol. 48, no. 8, pp. 1527-1537, 2006.

[3] R. M. Carney, K. E. Freedland, and A. S. Jaffe, "Depression screening in patients with heart disease," JAMA, vol. 301, no. 13, pp. 1337-1338, 2009.

[4] A. Newhouse and W. Jiang, "Heart failure and depression," Heart Failure Clinics, vol. 10, no. 2, pp. 295-304, 2014.

[5] P. Ponikowski, A. A. Voors, S. D. Anker et al., "2016 ESC guidelines for the diagnosis and treatment of acute and chronic heart failure: the task force for the diagnosis and treatment of acute and chronic heart failure of the European Society of Cardiology (ESC) developed with the special contribution of the Heart Failure Association (HFA) of the ESC," European Heart Journal, vol. 37, no. 27, pp. 2129-2200, 2016.

[6] C. W. Yancy, M. Jessup, B. Bozkurt et al., "2013 ACCF/AHA guideline for the management of heart failure: executive summary: a report of the American College of Cardiology Foundation/American Heart Association task force on 
practice guidelines," Circulation, vol. 128, no. 16, pp. 1810-1852, 2013.

[7] A. Bo, W. Mao, and M. A. Lindsey, "Effects of mind-body interventions on depressive symptoms among older Chinese adults: a systematic review and meta-analysis," International Journal of Geriatric Psychiatry, vol. 32, no. 5, pp. 509-521, 2017.

[8] P. Cabral, H. B. Meyer, and D. Ames, "Effectiveness of yoga therapy as a complementary treatment for major psychiatric disorders: a meta-analysis," The Primary Care Companion for CNS Disorders, vol. 13, no. 4, p. 10r01068, 2011.

[9] F. Wang, E.-K. O. Lee, T. Wu et al., "The effects of tai chi on depression, anxiety, and psychological well-being: a systematic review and meta-analysis," International Journal of Behavioral Medicine, vol. 21, no. 4, pp. 605-617, 2014.

[10] C. Wang, R. Bannuru, J. Ramel, B. Kupelnick, T. Scott, and C. H. Schmid, "Tai Chi on psychological well-being: systematic review and meta-analysis," BMC Complementary and Alternative Medicine, vol. 10, no. 1, p. 23, 2010.

[11] J. O. Younge, R. A. Gotink, C. P. Baena, J. W. Roos-Hesselink, and M. M. Hunink, "Mind-body practices for patients with cardiac disease: a systematic review and meta-analysis," $E$ ropean Journal of Preventive Cardiology, vol. 22, no. 11, pp. 1385-1398, 2015.

[12] R. A. Gary, S. B. Dunbar, M. K. Higgins, D. L. Musselman, and A. L. Smith, "Combined exercise and cognitive behavioral therapy improves outcomes in patients with heart failure," Journal of Psychosomatic Research, vol. 69, no. 2, pp. 119-131, 2010.

[13] C. Lan, S. Y. Chen, J. S. Lai, and A. M. Wong, "Tai Chi Chuan in medicine and health promotion," Evidence-Based Complementary and Alternative Medicine, vol. 2013, Article ID 502131, 17 pages, 2013.

[14] T. C. Koh, "Qigong-Chinese breathing exercise," The American Journal of Chinese Medicine, vol. 10, no. 1-4, pp. 86-91, 1982.

[15] Y. Guo, H. Shi, D. Yu, and P. Qiu, "Health benefits of traditional Chinese sports and physical activity for older adults: a systematic review of evidence," Journal of Sport and Health Science, vol. 5, no. 3, pp. 270-280, 2016.

[16] G. E. Ibañez, K. Fennie, and L. Larkey, "Qigong as a promising mind-body exercise for cognitive functioning: letter to editor," Journal of the American Geriatrics Society, vol. 67, no. 7, pp. 1533-1534, 2019.

[17] W. W. Y. So, S. Cai, S. Y. Yau, and H. W. H. Tsang, "The neurophysiological and psychological mechanisms of Qigong as a treatment for depression: a systematic review and metaanalysis," Frontiers in Psychiatry, vol. 10, p. 820, 2019.

[18] J. Kong, G. Wilson, J. Park, K. Pereira, C. Walpole, and A. Yeung, "Treating depression with tai chi: state of the art and future perspectives," Frontiers in Psychiatry, vol. 10, p. 237, 2019.

[19] J. M. Qu and X. Q. Wang, Traditional Chinese Exercises, Cambridge Scholars Publishing, Newcastle-Upon-Tyne, UK, 2015.

[20] I. Chi, M. Jordan-Marsh, M. Guo, B. Xie, and Z. Bai, “Tai chi and reduction of depressive symptoms for older adults: a meta-analysis of randomized trials," Geriatrics \& Gerontology International, vol. 13, no. 1, pp. 3-12, 2013.

[21] J. Yin and R. K. Dishman, "The effect of Tai Chi and Qigong practice on depression and anxiety symptoms: a systematic review and meta-regression analysis of randomized controlled trials," Mental Health and Physical Activity, vol. 7, no. 3, pp. 135-146, 2014.
[22] F. Wang, J. K. Man, E. K. Lee et al., "The effects of qigong on anxiety, depression, and psychological well-being: a systematic review and meta-analysis," Evidence-Based Complementary and Alternative Medicine, vol. 2013, Article ID 152738, 16 pages, 2013.

[23] D. Cheng, B. Wang, Q. Li, Y. Guo, and L. Wang, "Research on function and mechanism of Tai Chi on cardiac rehabilitation," Chinese Journal of Integrative Medicine, vol. 26, no. 5, pp. 393-400, 2020.

[24] X. Q. Wang, Y. L. Pi, P. J. Chen et al., “Traditional Chinese exercise for cardiovascular diseases: systematic review and meta-analysis of randomized controlled trials," Journal of the American Heart Association, vol. 5, no. 3, p. e002562, 2016.

[25] W. Wang, M. Sawada, Y. Noriyama et al., "Tai Chi exercise versus rehabilitation for the elderly with cerebral vascular disorder: a single-blinded randomized controlled trial," Psychogeriatrics, vol. 10, no. 3, pp. 160-166, 2010.

[26] G. L. Yuan, H. B. Zhang, F. Zhou et al., "Effect of Taijiquan on depression, sleep quality and quality of life in elderly patients with chronic congestive heart failure and depression," Guangxi Medicine, vol. 38, no. 11, pp. 1547-1550, 2016, in Chinese.

[27] X. D. Deng, D. M. Yu, and X. L. Zhang, "Effects of Taijiquan exercise on cardiac function and psychology of patients with cardiac insufficiency after acute myocardial infarction," Journal of North Sichuan Medical College, vol. 33, no. 4, pp. 545-547, 2018, in Chinese.

[28] J. H. Cheng, Y. J. Wang, S. S. Chou et al., "Chan-Chuang Qigong improves exercise capacity, depression, and quality of life in patients with heart failure," $\mathrm{Hu} \mathrm{Li} \mathrm{Za} \mathrm{Zhi,} \mathrm{vol.} \mathrm{65,} \mathrm{no.} \mathrm{5,}$ pp. 34-44, 2018.

[29] D. E. Barrow, A. Bedford, G. Ives, L. O’Toole, and K. S. Channer, "An evaluation of the effects of Tai Chi Chuan and Chi Kung training in patients with symptomatic heart failure: a randomised controlled pilot study," Postgraduate Medical Journal, vol. 83, no. 985, pp. 717-721, 2007.

[30] L. S. Redwine, M. Tsuang, A. Rusiewicz et al., "A pilot study exploring the effects of a 12-week T'ai Chi intervention on somatic symptoms of depression in patients with heart failure," The Journal of Alternative and Complementary Medicine, vol. 18, no. 8, pp. 744-748, 2012.

[31] G. Y. Yeh, E. P. McCarthy, P. M. Wayne et al., "Tai Chi exercise in patients with chronic heart failure: a randomized clinical trial," Archives of internal medicine, vol. 171, no. 8, pp. 750-757, 2011.

[32] G. Y. Yeh, M. J. Wood, P. M. Wayne et al., "Tai Chi in patients with heart failure with preserved ejection fraction," Congestive Heart Failure, vol. 19, no. 2, pp. 77-84, 2013.

[33] L. S. Redwine, K. Wilson, M. A. Pung et al., "A randomized study examining the effects of mild-to-moderate group exercises on cardiovascular, physical, and psychological wellbeing in patients with heart failure," Journal of Cardiopulmonary Rehabilitation and Prevention, vol. 39, no. 6, pp. 403-408, 2019.

[34] D. Moher, A. Liberati, J. Tetzlaff, and D. G. Altman, "Preferred reporting items for systematic reviews and meta-analyses: the PRISMA statement," BMJ, vol. 339, p. b2535, 2009.

[35] J. P. T. Higgins and S. Green, Cochrane Handbook for Systematic Reviews of Interventions, John Wiley \& Sons, Hoboken, NJ, USA, January 2018, http://www.handbook. cochrane.org.

[36] X. Chen, G. Savarese, Y. Cai et al., "Tai Chi and Qigong practices for chronic heart failure: a systematic review and meta-analysis of randomized controlled trials," Evidence- 
Based Complementary and Alternative Medicine, vol. 2020, Article ID 2034625, 15 pages, 2020.

[37] M. Richardson, P. Garner, and S. Donegan, "Interpretation of subgroup analyses in systematic reviews: a tutorial," Clinical Epidemiology and Global Health, vol. 7, no. 2, pp. 192-198, 2019.

[38] Q. Gu, S.-J. Wu, Y. Zheng et al., "Tai Chi exercise for patients with chronic heart failure: a meta-analysis of randomized controlled trials," American Journal of Physical Medicine \& Rehabilitation, vol. 96, no. 10, pp. 706-716, 2017.

[39] A. H. Fischer, P. M. Rodriguez Mosquera, A. E. M. van Vianen, and A. S. R. Manstead, "Gender and culture differences in emotion," Emotion, vol. 4, no. 1, pp. 87-94, 2004.

[40] R.-H. Tu, Z.-Y. Zeng, G.-Q. Zhong et al., "Effects of exercise training on depression in patients with heart failure: a systematic review and meta-analysis of randomized controlled trials," European Journal of Heart Failure, vol. 16, no. 7, pp. 749-757, 2014.

[41] K. N. Bhatt, A. P. Kalogeropoulos, S. B. Dunbar, J. Butler, and V. V. Georgiopoulou, "Depression in heart failure: can PHQ-9 help?" International Journal of Cardiology, vol. 221, pp. 246-250, 2016.

[42] C. E. Angermann, G. Gelbrich, S. Störk et al., "Effect of escitalopram on all-cause mortality and hospitalization in patients with heart failure and depression: the MOOD-HF randomized clinical trial," JAMA, vol. 315, no. 24, pp. 2683-2693, 2016.

[43] J. A. Blumenthal, M. A. Babyak, C. O'Connor et al., "Effects of exercise training on depressive symptoms in patients with chronic heart failure: the HF-ACTION randomized trial," JAMA, vol. 308, no. 5, pp. 465-74, 2012.

[44] C. M. O'ConnorW. Jiang et al., "Safety and efficacy of sertraline for depression in patients with heart failure: results of the SADHART-CHF (sertraline against depression and heart disease in chronic heart failure) trial," Journal of the American College of Cardiology, vol. 56, no. 9, pp. 692-699, 2010.

[45] M. Ceccarini, G. M. Manzoni, and G. Castelnuovo, "Assessing depression in cardiac patients: what measures should be considered?" Depression Research and Treatment, vol. 2014, Article ID 148256, 17 pages, 2014.

[46] K. Lahlou-Laforêt, F. Ledru, R. Niarra, and S. M. Consoli, "Validity of beck depression inventory for the assessment of depressive mood in chronic heart failure patients," Journal of Affective Disorders, vol. 184, pp. 256-260, 2015.

[47] K. J. Miller, D. C. Gonçalves-Bradley, P. Areerob, D. Hennessy, C. Mesagno, and F. Grace, "Comparative effectiveness of three exercise types to treat clinical depression in older adults: a systematic review and network meta-analysis of randomised controlled trials," Ageing Research Reviews, vol. 58, p. 100999, 2020.

[48] K. F. Schulz, D. G. Altman, D. G. Altman, and D. Moher, "CONSORT 2010 statement: updated guidelines for reporting parallel group randomised trials," BMC Medicine, vol. 8, no. 1, p. 18, 2010.

[49] 2020, https://www.equator-network.org/?post_type=eq_ guidelines\&eq_guidelines_study_design=0\&eq_guidelines_ clinical_specialty=0\&eq_guidelines_report_section $=0 \& s=$ +CONSORT+extension\&btn_submit=Search+Reporting+ Guidelines. 